\title{
Charge Conjugation Violation in Supernovae and The Neutron Shortage for R-Process Nucelosynthesis
}

\author{
C. J. Horowitz and Gang Li \\ Nuclear Theory Center and Dept. of Physics, Indiana University, Bloomington, IN 47405
}

\begin{abstract}
Core collapse supernovae are dominated by energy transport from neutrinos. Therefore, some supernova properties could depend on symetries and features of the standard model weak interactions. The cross section for neutrino capture is larger than that for antineutrino capture by one term of order the neutrino energy over the nucleon mass. This reduces the ratio of neutrons to protons in the $\nu$-driven wind above a protoneutron star by approximately $20 \%$ and may significantly hinder r-process nucleosynthesis.
\end{abstract}

Core collapse supernovae are perhaps the only present day large systems dominated by the weak interaction. They are so dense that photons and charged particles diffuse very slowly. Therefore energy transport is by neutrinos (and convection).

We beleive it may be useful to try and relate some supernova properties to the symmetries and features of the standard model weak interaction. Parity violation in a strong magnetic field could lead to an asymmetry of the explosion [1]. Indeed, supernovae explode with a dipole asymmetry of order one percent in order to produce the very high 'recoil' velocities observed for neutron stars [2]. However, calculating the expected asymmetry from $\mathrm{P}$ violation has proved complicated. Although explicit calculations have yielded somewhat small asymmetries [3-5] it is still possible that more efficient mechanisms will be found.

In this paper we calculate effects of charge conjugation, C, violation in the Standard Model on the difference between neutrino and antineutrino interactions. In Quantum Electrodynamics $\mathrm{C}$ symmetry insures the cross section for $e^{-} p$ is equal to that for $e^{+} p$ scattering (to lowest order in $\alpha$ ). In contrast, the standard model has large parity, $\mathrm{P}$, and $\mathrm{C}$ violations (since the product $\mathrm{CP}$ is approxamitely conserved). Therefore the $\bar{\nu}$-nucleon cross sections are systematically smaller than $\nu$-nucleon cross sections.

However at the low $\nu$ energies in supernovae, time reversal symmetry limits the difference between $\nu$ and $\bar{\nu}$ cross sections. Time reversal can relate $\nu-N$ elastic scattering and $\bar{\nu}-N$ where the nucleon scatters from final momentum $p_{f}$ to initial 
momentum $p_{i}$. If the nucleon does not recoil then the $\nu$ and $\bar{\nu}$ cross sections are equal. Thus the difference between $\nu$ and $\bar{\nu}$ cross sections are expected to be of recoil order $E / M$ where $E$ is the neutrino energy and $M$ the nucleon mass. This ratio is relatively small in supernovae. However the coefficient multiplying $E / M$ involves the large weak magnetic moment of the nucleon (see below).

The standard model has larger $\nu$ cross sections than those for $\bar{\nu}$. For neutral currents, this leads to a longer mean free path for $\bar{\nu}_{x}$ compared to $\nu_{x}$ (with $\mathrm{x}=\mu$ or $\tau$ ). Thus even though $\nu_{x}$ and $\bar{\nu}_{x}$ are produced in pairs, the antineutrinos escape faster leaving the star neutrino rich. The muon and tau number for the protoneutron star in a supernova could be of order $10^{54}$ [6]. Supernovae may be the only known systems with large $\mu$ and or $\tau$ number. For charged currents, the interaction difference can change the equilibrium ratio of neutrons to protons and may have important implications for nucleosynthesis. We discuss this below. To our knowledge, all previous work on nucleosynthesis in supernovae assumed equal $\nu$ and $\bar{\nu}$ interactions (aside from the n-p mass difference).

The neutrino driven wind outside of a protoneutron star is an attractive site for r-process nucleosynthesis [7]. Here nuclei rapidly capture neutrons from a low density medium to produce heavy elements [8]. This requires, as a bare minimum, that the initial material have more neutrons than protons. The ratio of neutrons to protons $\mathrm{n} / \mathrm{p}$ in the wind depends on the rates for the two reactions:

$$
\begin{aligned}
& \nu_{e}+n \rightarrow p+e^{-}, \\
& \bar{\nu}_{e}+p \rightarrow n+e^{+} .
\end{aligned}
$$

The standard model cross sections for Eqs. (1a,1b) to order $E / M$ are,

$$
\sigma=\frac{G^{2} \cos ^{2} \theta_{c}}{\pi}\left(1+3 g_{a}^{2}\right) E_{e}^{2}\left[1-\gamma \frac{E}{M} \pm \delta \frac{E}{M}\right]
$$

with $G$ the Fermi constant (and $\theta_{c}$ the Cabbibo angle), $E_{e}=E \pm \Delta$ the energy of the charged lepton and $\Delta=1.293 \mathrm{MeV}$ is the neutron-proton mass difference. The plus sign is for Eq. (1a) and the minus sign for Eq. (1b). We use $g_{a} \approx 1.26$. Equation (2) neglects small corrections involving the electron mass and coulomb effects while the finite nucleon size only enters at order $(E / M)^{2}$.

We refer to the $\gamma$ term as a recoil correction. It is the same for $\nu$ and $\bar{\nu}, \gamma=$ $\left(2+10 g_{a}^{2}\right) /\left(1+3 g_{a}^{2}\right) \approx 3.10$. Finally, the $\delta$ term, $\delta=4 g_{a}\left(1+2 F_{2}\right) /\left(1+3 g_{a}^{2}\right) \approx 4.12$, involves the interference of vector $\left(1+2 F_{2}\right)$ and axial $\left(g_{a}\right)$ currents. This violates $\mathrm{P}$, which by $\mathrm{CP}$ invariance also violates $\mathrm{C}$. This increases the $\nu$ and decreases the $\bar{\nu}$ cross section, Note, $F_{2}$ is the isovector anomalous moment of the nucleon. (This is the weak magnetism contribution.)

The equilibrium electron fraction per baryon $Y_{e}$ (which is equal to the proton fraction assuming charge neutrality) is simply related to the rate $\bar{\lambda}$ for Eq. (1b) divided by the rate $\lambda$ for Eq. (1a).

$$
Y_{e}=\left(1+\frac{\bar{\lambda}}{\lambda}\right)^{-1}
$$


The ratio neutrons to protons is, $\frac{n}{p}=\frac{1}{Y_{e}}-1$.

In ref. [9] we calculate the reaction rates by averaging Eq. (2) over neutrino spectra to get,

$$
Y_{e}=\left(1+\frac{L_{\bar{\nu}_{e}} \bar{\epsilon}}{L_{\nu_{e}} \epsilon} Q C\right)^{-1} .
$$

Here $\epsilon(\bar{\epsilon})$ is the $\nu_{e}\left(\bar{\nu}_{e}\right)$ mean energy, $L_{\nu_{e}}\left(L_{\bar{\nu}_{e}}\right)$ is the $\nu_{e}\left(\bar{\nu}_{e}\right)$ luminosity, $Q$ is the correction from the reaction $\mathrm{Q}$ value,

$$
Q=\frac{1-2 \frac{\Delta}{\bar{\epsilon}}+a_{0} \frac{\Delta^{2}}{\bar{\epsilon}^{2}}}{1+2 \frac{\Delta}{\epsilon}+a_{0} \frac{\Delta^{2}}{\epsilon^{2}}},
$$

and with $\mathrm{C}$ violating one has a factor $C$,

$$
C=\frac{1-(\delta+\gamma) a_{2} \frac{\bar{\epsilon}}{M}}{1+(\delta-\gamma) a_{2} \frac{\epsilon}{M}} .
$$

Simply evaluating Eq. (6) for typical parameters yields $C \approx 0.8$. Thus, the difference between $\nu$ and $\bar{\nu}$ interactions reduces the equilibrium $n / p$ ratio by approximately $20 \%$. This is an important result and will be discussed below.

Evaluating Eq. (4) for the neutrino fluxes of a Supernova simulation by Wilson [10] shows that with the $C$ term the neutrino driven wind starts out proton rich and ends up with about equal numbers of neutrons and protons. When $\mathrm{C}$ violation is included the wind is never significantlt neutron rich. It is very unlikely that successful r-process nucleosynthesis can take place in the wind of this or similar models.

With the approximately $20 \%$ reduction in $\mathrm{n} / \mathrm{p}$ from the difference between $\nu$ and $\bar{\nu}$ interactions, there appears to be very serious problems with r-process nucleosynthesis in the wind of present supernova models. In addition to the initial lack of neutrons, one has to overcome the effects of neutrino interactions during the assembly of $\alpha$ particles and during the r-process itself [11]. These further limit the available neutrons per seed nucleus. Thus, it is unlikely that present wind models will produce a successful r-process. Of course, the wind in supernovae may not be the $\mathrm{r}$-process site, although this may be unappealing (see for example $[8,12]$ ). If the wind is not the site, one must look for alternative environments.

However, the effects of neutrino interactions may be very general. The only requirement is that energy transport from neutrinos plays some role in helping material out of a deep gravitational well. Given this, it is quite likely that the $\mathrm{n} / \mathrm{p}$ ratio will be determined by the relative rates of Eqs. (1a,1b). Therefore differences in $\nu$ and $\bar{\nu}$ interactions may be important for just about any nucleosynthesis site that involves neutrinos.

If the $\nu$-driven wind is the r-process site, it is very likely, present models of the neutrino radiation in supernovae are incomplete. The high values of $Y_{e}$ make it almost impossible to have a successful r-process by only changing matter properties, such as the entropy. The neutrino fluxes will (almost assuredly) need to be changed. 
Changes in the astrophysics used in the simulations or new neutrino physics such as neutrino oscillations [13] could change $\bar{\epsilon}, \epsilon$ and or the luminosities and lead to a more neutron rich wind. The oscillations of more energetic $\bar{\nu}_{x}$ with $\bar{\nu}_{e}$ could increase $\bar{\epsilon}$. However, we have some information on the $\bar{\nu}_{e}$ spectrum from SN1987a [14]. Thus one can not increase $\bar{\epsilon}$ without limit. Indeed if anything, the Kamiokande data suggest a lower $\bar{\epsilon}$. Any model which tries to solve r-process nucleosynthesis problems by increasing $\bar{\epsilon}$ should first check consistency with SN1987a observations [15]. Alternative modifications could include oscillations of $\nu_{e}$ to a sterile neutrino or a lowering of $\epsilon$. (However, we know of no model which lowers $\epsilon$.) Whatever the modification of the neutrino fluxes, one will still need to include the differences between $\nu$ and $\bar{\nu}$ interactions in order to accurately calculate $\mathrm{n} / \mathrm{p}$.

In conclusion, supernovae are one of the few large systems dominated by energy transport from weakly interacting neutrinos. Therefore, some supernova properties may depend on symmetries and features of the standard model weak interactions. The cross secton for neutrino capture is larger than that for antineutrino capture by a term of order the neutrino energy over the nucleon mass. This difference between neutrino and antineutrino interactions reduces the ratio of neutrons to protons in the $\nu$-driven wind above a protoneutron star by approximately $20 \%$ and may significantly hinder r-process nucleosynthesis.

This work was supported in part by DOE grant: DE-FG02-87ER40365.

\section{REFERENCES}

1. A. Vilenkin, 1979, unpublished; Ap J 451 (1995) 700. N. N. Chugai, Pisma Astron. Zh. 10 (1984) 210. [Sov. Astron. Lett. 10 (1984) 87.]

2. A. G. Lyne and D. R. Lorimer, Nature 369 (1994) 127.

3. C. J. Horowitz and J. Piekarewicz, Nuc. Phys. A640 (1998)281.

4. C.J. Horowitz and Gang Li, Phys. Rev. Lett. 80 (1998) 3694, Erratum-ibid. 81 (1998) 1985.

5. Phill Arras and Dong Lai, astro-ph/9806285.

6. C.J. Horowitz and Gang Li, Phys. Lett. B443 (1998) 58.

7. S.E. Woosley and R.D. Hoffman, ApJ. 395 (1992) 202. B.S. Meyer, W.M. Howard, G.J. Mathews, S. E. Woosley and R.D. Hoffman, ApJ. 399 (1992) 656.

8. B. S. Meyer, Ann. Rev. Astron. Astrophys. 32 (1994) 153.

9. C. J. Horowitz and Gang Li, Phys.Rev.Lett. 82 (1999) 5198.

10. Y.-Z. Qian and S.E. Woosley, ApJ. 471 (1996) 471.

11. B.S. Meyer, ApJL. 449 (1995) 792. G. M. Fuller and B.S. Meyer, ApJ 453 (1995) 792. B.S. Meyer, G. McLaughlin and G.M. Fuller, astro-ph/9809242.

12. J.J. Cowan, F.-K. Thielemann and J.W. Truran, Phys. Rep. 208(1991) 267.

13. Yong-Zhong Qian and G.M. Fuller, Phys. Rev. D52 (1995) 656.

14. K. Hirata et al., Phys. Rev. Lett. 58 (1987) 1490; R. M. Bionta et al., Phys. Rev. Lett. 58 (1987) 1494.

15. A. Yu. Smirnov, D. N. Spergeland and J.N. Bahcall, Phys Rev. D49 (1994) 1389. B. Jegerlehner, F. Neubig and G. Raffelt, Phys. Rev. D54 (1996) 1194. 